\title{
Prophylaxis against infective endocarditis
}

\author{
National Institute for Health and Clinical Excellence. \\ Prophylaxis against infective endocarditis: antimicrobial \\ prophylaxis against infective endocarditis in adults and children \\ undergoing interventional procedures. London: National Institute \\ for Health and Clinical Excellence; 2008:clinical guideline 64
}

Scope and purpose The guideline was developed to provide evidence-based recommendations to guide healthcare professionals in the appropriate care of people thought to be at risk of infective endocarditis who may require antimicrobial prophylaxis before an interventional procedure. Further details of the scope are available at www.nice.org. uk/nicemedia/pdf/Finalscope040707.pdf.

Methods The guideline was developed by a guideline development group consisting of 12 members and a National Institute for Health and Clinical Excellence (NICE) Short Clinical Guidelines Technical Team using methods described in the NICE guidelines manual (available from www. nice.org.uk/guidelinesmanual).

Review and updating Review and updating will take place in line with procedures outlined in the NICE guidelines manual.

Recommendations Recommendations were as follows:

1. Healthcare professionals should regard people with the following cardiac conditions as being at risk of developing infective endocarditis (IE):

- acquired valvular heart disease with stenosis or regurgitation

- valve replacement

- structural congenital heart disease, including surgically corrected or palliated structural conditions, but excluding isolated atrial septal defect, fully repaired ventricular septal defect or fully repaired patent ductus arteriosus, and closure devices that are judged to be endothelialised

- previous IE

- hypertrophic cardiomyopathy

2. Healthcare professionals should offer people at risk of IE clear and consistent information about prevention, including:

- the benefits and risks of antibiotic prophylaxis, and an explanation of why antibiotic prophylaxis is no longer routinely recommended

- the importance of maintaining good oral health

- symptoms that may indicate IE and when to seek expert advice

- the risks of undergoing invasive procedures, including nonmedical procedures such as body piercing or tattooing
Address for correspondence: National Institute for Health and Clinical Excellence, MidCity Place, 71 High Holborn, London WC1V 6NA, UK.

E-mail: nice@nice.org.uk
3. Antibiotic prophylaxis against IE is not recommended:

- for people undergoing dental procedures

- for people undergoing nondental procedures at the following sites: upper and lower gastrointestinal tract; genitourinary tract (includes urological, gynaecological and obstetric procedures, and childbirth); and upper and lower respiratory tract (includes ear, nose and throat procedures and bronchoscopy)

4. Chlorhexidine mouthwash should not be offered as prophylaxis against IE to people at risk of IE when they undergo dental procedures.

5. Any episodes of infection in people at risk of IE should be investigated and treated promptly to reduce the risk of endocarditis developing.

6. If a person at risk of IE is receiving antimicrobial therapy because they are undergoing a gastrointestinal or genitourinary procedure at a site where there is a suspected infection, the person should receive an antibiotic whose spectrum covers organisms that cause IE.

Research recommendations IE is a rare condition and UK research in this area would be facilitated by the availability of a national register of IE cases that could offer data into the 'case' arm of proposed casecontrol studies. Two specific areas of required research were highlighted. First, the risk of developing IE in people who have acquired valvular disease and structural congenital heart disease should be investigated using a population-based cohort study design to allow direct comparison between groups and estimation of both relative and absolute risk. Second, the frequency and level of bacteraemia caused by nonoral daily activities (for example, urination or defecation) requires research to determine quantitatively the frequency and level of bacteraemia.

\section{Commentary}

As noted in the press release from NICE accompanying the launch of this latest guidance, it represents a significant change to clinical practice for the UK. It has been accepted practice for decades to use antibiotic prophylaxis to prevent IE, a rare condition with significant mortality and morbidity and an annual incidence of 10 per 100,000 people. Rates are higher in high-risk groups such as injecting drug users, a group not in fact dealt with in these recommendations.

The guideline has been developed using robust and well-established methods and a significant amount of detailed additional material is available from the NICE website (www.nice.org.uk/guidance/index.jsp?action=byID\&o=11938). The clear, unequivocal nature of this guidance and the new recommendations have been warmly welcomed by the dental profession at large within the UK. Previous guidelines from the British Society for Antimicrobial Chemotherapy $^{1}$ and the American Heart Association ${ }^{2}$ had challenged the existing dogma which saw empirical prescription of antibiotic prophylaxis for a wide range of cardiac conditions. The latter two guidelines highlight the prevalence of bacteraemias that arise from everyday activities such as toothbrushing, the lack of association between episodes of IE and prior interventional 
procedures, and the lack of efficacy of antibiotic prophylaxis regimens. Both continued to recommend prophylaxis for some cardiac conditions, however.

This detailed guidance was developed by a team including cardiologists and cardiac surgeons, microbiologists, pharmacists, dental practitioners, paediatric dentists and academic dentists as well as two patient representatives and co-opted experts in gastroenterology, obstetrics, urology, otolaryngology, respiratory medicine and anaesthetics. It fully explores the existing evidence on effectiveness, risks and cost-effectiveness, and fully justify the rationale for their clear and unequivocal recommendations.

I feel that the challenge will come when implementing these recommendations: it is a paradigm shift in clinical practice for both patients and practitioners which may engender resistance from some, particularly in the light of differing recommendations from the previous, recent, guidelines. ${ }^{1,2}$ To assist patients, NICE have produced a clear patient information sheet. There is also the usual NICE quick reference guide and some further information to assist with local implementation including a set of PowerPoint (Microsoft, Redmond, Washington, USA) slides outlining the main elements of the guidance.

\section{Derek Richards}

Centre for Evidence-based Dentistry, Oxford, UK.

1. Gould FK, Elliott TSJ, Foweraker J, et al. Guidelines for the prevention of endocarditis: report of the Working Party of the British Society for Antimicrobial Chemotherapy. J Antimicrob Chemother 2006; 57:1035-1042.

2. Wilson W, Taubert K, Gewitz M, et al. Prevention of infective endocarditis. Guidelines from the American Heart Association: a guideline from the American Heart Association Rheumatic Fever, Endocarditis, and Kawasaki Disease Committee, Council on Cardiovascular Disease in the Young, and the Council on Clinical Cardiology, Council on Cardiovascular Surgery and Anesthesia, and the Quality of Care and Outcomes Research Interdisciplinary Working Group. Circulation 2007; 116:1736-1754.

Evidence-Based Dentistry (2008) 9, 37-38. doi:10.1038/sj.ebd.6400577 